\title{
HUMAN-COMPUTER INTERACTION AND USER EXPERIENCE IN SMART HOME RESEARCH: A CRITICAL ANALYSIS
}

\author{
Yu Zhao, University of North Alabama,yzhao8@una.edu \\ Xihui Zhang, University of North Alabama,xzhang6@una.edu \\ John Crabtree, University of North Alabama, jcrabtree@una.edu
}

\begin{abstract}
The development of the smart home presents new challenges to the application of human-computer interaction and user experience (HCI/UX), providing many possibilities for smart home users to suggest additional practical requirements. Based on diverse fundamental theories and various practical implementations, we propose four innovative approaches including efficient general control panels, effective user interfaces, variable accessibility, and secure privacy. These proposed approaches can help designers and developers optimize the user experience in smart homes and satisfy the users' non-functional requirements by improving the efficiency and effectiveness of the user experience. We discuss the importance of each of these approaches and provide detailed instructions on how to implement each of them. Finally, we conclude the paper with an analysis of the practical implications of our approaches and a discussion of possible new features of the smart home.
\end{abstract}

Keywords: Smart Home, Technology, User Experience, Human-Computer Interaction

\section{INTRODUCTION}

A home is one of the essential elements of modern life. Today, the traditional concept of the home has been enriched to be more colorful and diversified by modern information technology. The smart home was first mentioned in the 1990's (Harper, 2003) and according to the Smart Home Association, the smart home is "the integration of technology and services through home networking for a better quality of living” (Roe, 2007, p. 110).

The design of the smart home is based on analyzing the fundamental requirements for modern living, which include construction, comfort, Internet communication, digital electronics, and automated devices. The concept of the smart home combines commercial products with system services and management in order to support additional requirements in the areas of efficiency, intelligence, security, and interactivity applied in an eco-friendly fashion. These requirements reflect a new trend of expectations for living conditions that have evolved with the development of our modern society. Smart home products have recently seen an increase in published studies and innovative applied practice, making the smart home a fast-developing and popular area of research.

In recent years, the development of the information society and advances in environmental intelligence have led to the rapid growth of system-based applications and services, which has brought a variety of new human-computer interaction (HCI) dimensions into play. Intelligent environments enhance the importance of HCI in the information society due to the appearance of new forms of applications that can provide for our daily needs in all sorts of environments and application domains. According to a report by the European Commission for Information Society and Media, the concept of environmental intelligence provides a new perspective for emphasizing a higher level of user-friendly interaction, more efficient service support, more robust user authority, and increased support for HCI best practices (Roe, 2007). In an intelligent environment, people are exposed to different kinds of objects and conditions, in which the environment is able to identify and react to in a seamless and transparent way. The smart home is becoming an important application field in the modern information society. There are many new digital products and services being developed in support of these integrated and intelligent computing environments, which in turn present new challenges to smart home development and the application of HCI best practices. 


\section{Issues in Information Systems \\ Volume 17, Issue III, pp. 11-19, 2016}

In our modern society, people tend to prefer simplicity. The development of the smart home caters to the requirements for ease of living while providing a new and revolutionized lifestyle. First of all, the smart home can maximize family time by simplifying the operation of electronic appliances. Universal control pads can be used to operate a variety of smart devices such as TVs, computers, air conditioners, and lights. We can save time by operating such devices via a smart home control system so that we can spend more time with our family and friends. In addition, a smart home can be highly customized according to personal preferences. For example, users can change the color of lights or the content of the digital wallpaper whenever they want to with only one or two clicks. We can also create distinct themes or styles in different rooms more easily in a smart home. Finally, the smart home can produce a more secure environment for the family. With the advances in monitoring and notification systems, we can check our home conditions using mobile devices while being away from home. The smart home can also help kids or disabled people lock or unlock the doors and windows as well as remind the elderly to turn off the stove or lights. In short, the smart home can significantly improve the quality of life.

To support the various requirements of the smart home, the development of digital products and user interfaces is essential. New product features and services can attract the attention of millions of users. As a result, user experience research has become an integral part of the product design process, which in turn plays an essential role in product innovation. An effective user interface plays a critical role in making the smart home more reliable and enjoyable. First of all, users need a centralized control system in which the user interface for each smart home device could potentially be displayed in a device-specific fashion. For example, the user interface for an air conditioner should be different from that for an oven. In addition, an effective HCI design is required to guide users in the proper operation of the various smart home devices. The system should remember selected options and give proper feedback in response to invalid selections. Effective HCI that meets the users' needs enhances the user experience, which in turn improves the quality of life in a smart home.

\section{LITERATURE REVIEW}

The smart home, also known as the e-home, combines the concepts of the Internet of Things, human-computer interaction, and a variety of modern information technologies, in order to provide an intelligent environment that gives users more control (Davidoff et al., 2006) and enhances the quality of their lives. The demand for intelligent products is increasing significantly, especially with the commoditization of the smart phone and its inherent usercentered emphasis. The best way to maximize the intelligence of a smart home is to optimize the human-computer interaction system. First of all, it is important to understand the characteristics of the occupants of the smart home. These users of the smart home products and services can vary from young to old, healthy to disabled, and experts to novices in using control devices such as handheld computers. It is therefore important for the smart home environment to be configurable to meet the needs of a diverse user base. For instance, a smart home could be designed to support elderly and/or disabled people who live by themselves via a combination of intelligent devices that assist them with their daily tasks while providing a safe and secure environment (Zhang et al., 2009).

\section{Smart Home for Healthcare}

As the population of older adults increases and healthcare resources struggle to keep pace, technological solutions are critical in improving the quality of life for the elderly. Many scientists believe that the smart home can achieve its full potential in providing health assistance for people in need (Bouchard et al., 2012). The primary goal of the smart home in healthcare is to provide a monitoring system that constantly generates unobtrusive data streams that can be used to assess the status of one's health. Moreover, "measures of physiological signs and behavioral patterns can be translated into accurate predictors of health risk, even at an early stage, and can be combined with alarmtriggering systems as a technical platform to initiate appropriate action" (Chan et al., 2009, p. 93). Although the smart home monitoring system is acceptable for older people according to the existing research, the perceived value from such systems is difficult to assess. It is therefore important to establish an effective feedback system for users associated to the health-related monitoring data. With effective feedback, the health monitoring system can promote better engagement with older people while improving the overall quality of the healthcare system (Le et al., 2014). 


\section{Issues in Information Systems \\ Volume 17, Issue III, pp. 11-19, 2016}

Besides the smart home requirements for the elderly, there have been an increasing number of requirements for features that support people with disabilities. For instance, people with dementia (e.g., memory loss) might need to live in a smart environment to compensate for their diminished abilities. Nevertheless, when we talk about the smart home or the smart environment, the functionality created through the use of high-end technologies are often complicated for the average end user to fully comprehend, let alone for people with dementia. It is therefore essential that devices are designed with a goal of minimizing direct, full-featured interactions with end users. The common smart home concepts focus on human-computer interactions and task completion. However, the smart home for people with dementia should focus on remembering users' behaviors, so that it can play the role of "reminder" in human care. In other words, the smart home features would act as household members, providing services such as turning devices on or off based on the circumstances of the situation. Of course, these technologies can hardly replace human care, but the smart home could provide some relief for the people providing healthcare services and improve the overall quality of healthcare (Orpwood et al., 2005).

\section{Smart Home for Energy Efficiency}

In the past few years, extensive research and analysis regarding home energy technologies have been conducted. Today, there are a variety of ways to reduce the energy cost in homes by using smart programs such as efficient energy management (De Silva et al., 2012). For example, users' preferences concerning the living environment can be monitored more efficiently by adding more sensors to improve the accuracy of monitoring. In addition, some research recommends the use of a multi-agent technique in order to reduce energy consumption. Improving energy efficiency for the smart home and proposing a comprehensive smart home system concept will not only minimize current smart home energy problems, but also improve the chances of creating a fully-functional smart home in the near future (De Silva et al., 2012).

\section{Interface Design and Human-computer Interaction}

With the rapid development of information technology, many ideas proposed in the past have become a reality. The use of the cloud computing services, the emphasis on the interface design, the theory of human-computer interaction, and the development of the Internet of Things together provide brand new opportunities for the advancement of the smart home. In order to build an effective cloud computing environment for the smart home, we need to overcome the main challenge, which is to manage and coordinate humans and objects in a smart home environment. One solution is for designers to create effective smart home devices according to user specifications, using a computeraided design interface, also recognized as a designer-oriented interface. To improve the efficiency of contact and communications between human and the artificial intelligence through the use of Internet infrastructure and applications, a computer-aided or designer-oriented interface is recommended in the creation of smart home devices (Chen et al., 2010).

In recent years, user interfaces have been considered a critical factor in satisfying the requirements and preferences of individual users, which result in user interfaces that more like a personal user interface. In order to develop an effective personal user interface, designers should consider context-driven development as an essential element in the creation of the user interface. Context-driven user interfaces play an increasingly important role in encouraging users to adapt to the smart home or the Ambient Assisted Living environment (Roe, 2007), and some techniques related to context-driven user interfaces such as dynamic approaches at runtime have also been proposed (Zimmermann et al., 2013).

Recent advances and technologies have increased smart home performance expectations. Technologies related to physical settings such as sensors and actuators provide an opportunity to optimize the functionality that is responsible for maximizing comfort within the smart home, while minimizing the consumption of resources (Rashidi \& Cook, 2009). The development of sensors and actuators has made it possible to monitor user behavior. The most recent research pays more attention to the interactions between humans and the system; as a result, a smart home equipped with multiple sensitive services provides an effective platform to meet most of the requirements for healthcare applications (Vega-Barbas et al., 2015). This advanced technology has made smart home devices more intuitive and provides a solid foundation to support a gesture-based interface. A gesture is "any physical movement that a digital system can sense and respond to without the aid of a traditional pointing device such as a mouse or 
stylus. A wave, a head nod, a touch, a toe tap, and even a raised eyebrow can be a gesture" (Saffer, 2009, p. 2). Gesture-based interfaces such as camera-based gestural interfaces and motion sensor-based gestural interfaces (Kühnel et al., 2011) can be used to recognize the physical movements of users without the intervention of traditional devices such as a keyboard or a mouse (Choi et al., 2014). Humans can interact with the surrounding environment in a number of ways. We can control different kinds of devices in specific ways, and we can act and react in different environmental conditions. Only if the environment can recognize our behaviors and respond to what we do, can we truly call it a smart home. It is therefore important to recognize that current trends have shifted toward the use of more intuitive user interfaces.

With the development of the smart environment concept from healthcare, information technologies, movement capture monitors, and effective user interfaces, the human-computer interaction can become a reality in our daily life. A smart home provides an intelligent environment equipped with various specific devices that can manipulate an ambient intelligence with a series of smart automatic controls. This intelligent environment detects and responds to human behaviors and provides a variety of services for residents (De Silva et al., 2012). Along with the advancement of the concepts and technologies of human-computer interaction and the development of modern society, research into the Internet of Things has increased over the years. Artificial psychology theory plays an increasingly essential role in the smart home related to the Internet of Things. The harmony of the smart home has been the focus of recent research studies. In order to improve the harmony of human-computer interaction, research studies of the emotional model and emotional recognition of human-computer interaction have also been conducted (Du et al., 2013).

\section{A Summary of Status}

"Smart homes are an advancing wave of technological development whose success depends on a coalescence between the visions of technology developers for enhanced functionality and energy management, and the needs and demands of households in the complex places that are homes" (Wilson et al., 2015, p. 11). In order to improve the user experience in the smart home, an effective and efficient user interface is required. A quality smart home system should be built not only upon advanced smart devices, but also upon an excellent HCI design. According to our studies, we believe that the following research objectives should also be accomplished. First of all, a central control system should be established and an operational pad is also required to integrate all kinds of smart devices. The operating systems of smart devices should be united, and standards of communication between different devices should be consistent, which can provide an essential foundation for designing user interfaces for the smart home. In addition, a general guide addressing how to design user interfaces for smart devices that are targeted for use in the smart home environment should be created. We think that user interfaces for different smart devices are unique. The design of the user interface should be developed based on main functions of smart devices and the style of design should also follow the basic theories of HCI. Furthermore, the user interface for every individual should be varied based on the accessibility needs of the user. User authorization and authentication capabilities are necessary to assist in providing a secure intelligent environment. Customizable interfaces for different users are also important to improve user experience within the smart home. Finally, a secure mechanism of information transfer should be built to ensure the safety of the user's data. The information and behavior of the user are easy to record in the smart home, and it is therefore essential to protect the user's privacy.

\section{FOUR APPROACHES TO IMPROVE HCI AND UX}

Faced with many challenges, the advancement of the smart home today remains slow. There are numerous technological bottlenecks that should be dealt with before we can expect the smart home to become a widespread reality. It is therefore critical to discuss objectives that can contribute to the successful implementation of the smart home. As such, we propose four approaches to improving the design of the HCI and ultimately the user experience within the smart home. 


\section{Issues in Information Systems \\ Volume 17, Issue III, pp. 11-19, 2016}

\section{Efficient General Control Panel}

In order to improve the HCI of the smart home, a general control panel is necessary. Today, we have a remote for almost every electronic device in our home such as TV, DVD player, and air conditioner, and it is increasingly difficult for us to distinguish these various remotes from each other by mere appearance. Dealing with multiple controllers is not a wise option in the smart home, therefore a universal controller should be developed. A great user experience depends on an excellent HCI design realized via the universal control panel. There are still some issues that need to be addressed before focusing on the control panel design. First of all, a united information communication standard should be established. We want our universal controller to manipulate all sorts of smart devices at the same time. It is very important to improve user experience and save time by simplifying the manipulation processes. If all the smart devices can be connected into one control panel, the operating systems of the control panel should also be standardized to run different user interfaces. An effective way to improve the user experience is by synchronizing user interfaces with the update of smart devices. For example, if you add a new air purifier to an existing air conditioner, the user interface of your controller should update the function at the same time. It is essential to establish integrated communication standards for smart devices before the implementation of the smart home; in other words, no matter what kind of smart device that users buy from smart home companies, the device should be compatible with the control panel. An efficient and effective user interface design can only be developed based on a mature operating system, and only if a proper standard is established, can an effective smart home be realized.

In addition, information exchange among smart devices should be compatible and standardized. Smart home devices are expected to be intelligent enough to communicate with each other and the information captured by smart devices as well as the records of users' operations should be shared among smart devices. In order to improve the quality of the user experience, we want our smart home to act as a friendly reminder by notifying us of what is happening in our home. For instance, if the user is watching TV while the oven is baking food, a notification about the oven will pop up on the TV screen when the food is ready. The ability to notify users when necessary plays an increasingly important role in providing an intelligent environment especially for those who have trouble remembering things as well as those who are disabled. The information exchange among smart devices provides a significant foundation for a higher level of HCI that is modeled as direct interaction with smart devices. It is also essential to establish a secure home environment when sharing data captured by multiple devices. In order to achieve efficient and effective information exchange, we could create a database that leverages cloud computing. Information about the user can be recorded by the control panel or smart devices on a regular, daily basis, and the use of cloud computing and storage in the cloud can provide a convenient integration point.

\section{Effective User Interface}

A user interface is an operational panel that can be employed to allow the user to manipulate functions and behaviors of different kinds of applications via simple movements. In a smart home, all sorts of smart devices can be operated by the control panel, which raises new challenges for user interface designers. An effective user interface plays a key role in improving user experience. The user interface is widely used by all sorts of common devices including electronic appliances, mobile devices, and social media websites. The development of HCI and its impact on the user interface play an increasingly significant role in building an effective smart home. In order to design an efficient and effective user interface, we propose the following guidelines.

Visualize the function. A user interface for a smart home is different from what we use on phones or websites. The functions of different smart devices are very important for users, and this is the reason why we purchase various electronic appliances for use in our homes. In order to improve the user experience for smart home owners, the functions of different smart devices should be visualized (see Figure 1). First of all, users need to instantly know which devices are being used. A virtual imitation of smart device functionality on the control panel is very helpful for users to recognize controls and their associated status. For instance, when we want to operate a stove in our kitchen, a model of the stove should be displayed surrounded by key functions such as temperature control, timers, and switches (see Figure 1a). It is more efficient to differentiate different devices via synchronous simulation models as opposed to a collection of buttons. Operating concentrated collections of buttons is not very intuitive and can leave users with a negative impression of the system as a whole. A simplified simulation model is also more 
appropriate for the elderly and for people with disabilities. Visualized models are essential in improving the user experience and, by extension, provide more positive experiences for the end users when compared with pressing buttons.

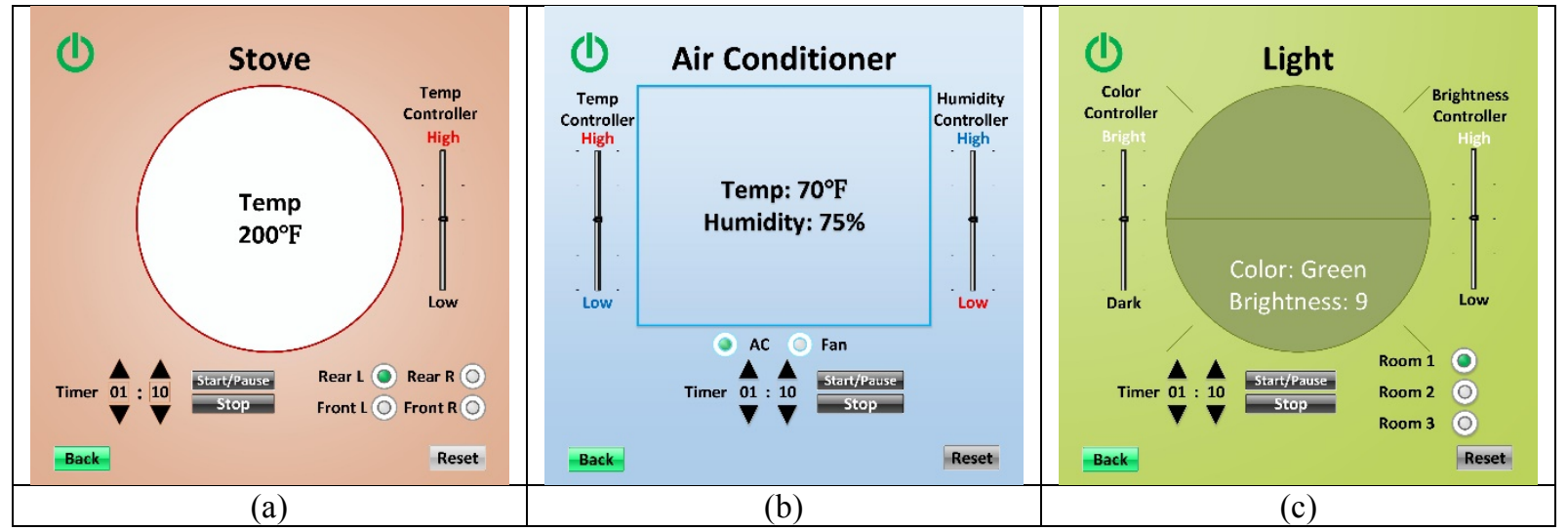

Figure 1. Visualized Models of Smart Devices

Provide efficient feedback. A quality HCI design cannot be achieved without efficient feedback. The feedback mechanism plays an important role in modern HCI design, and it has been widely used on a variety of user interface types such as websites, mobile applications, and operating systems to improve user experience. Intelligent devices used in the smart home should provide efficient feedback to the devices themselves or to the user's control panel. The feedback can be designed in multiple modes such as dialog boxes, lights, sounds, and vibrations. An efficient feedback system can provide various physical signals to enhance HCI, and this feedback is essential in reducing the user's operational mistakes. For instance, if the user inputs an invalid number or date in the control panel, the system should notify the user with a dialog or recommendation before continuing to proceed. Furthermore, the feedback system is also a foundational component in allowing the control panel to function as a reminder. An efficient feedback system also plays an essential role in improving the user experience for the elderly and the disabled. Older adults or people with disabilities need more efficient feedback to help them remember which operations have been completed and to require them to confirm critical operations before processing their commands.

\section{Variable Accessibility}

An effective user interface provides a general prototype for the control panel of the smart home. Nevertheless, the user experience, especially for the elderly, the disabled, and children, can be significantly improved through personalized accessibility. First of all, the user interface for people with disabilities should be varied according to their capabilities, and the user interface should also be configurable among different users. In order to enhance the overall user experience of the smart home, the design of the user interface should be as accessible as possible, especially for those with special needs. One of the main purposes of establishing a smart home is to assist users in improving the quality of life through accessible designs and facilities. A special user interface for the disabled can provide them with a simplified mode of operation and thus a better experience of HCI. It is important to understand that the disabled can accomplish a majority of operations at home with the help of a personalized user interface. For instance, the user interface could be switched to cater to different requirements. When one of your disabled friends wants to visit your smart home, you should be able to easily switch the user interface to a particular mode to allow all kinds of smart devices to be controlled in a different way. If certain users cannot move their fingers to touch the screen, they may be able to speak to the control panel. In order for them to do this, however, the user interface mode should be changed from a touchable, visualized interface into a voice controlled interface. Users can employ a sequential list of words or phrases to control the functions of the smart devices. For example, if a user with disability wants to turn on the air conditioner (see Figure 1b), he or she just needs to say "air conditioner," "turn on," "temperature," then "up" or "down." It is important for many users with physical disabilities to be able to use voice commands that are usually accomplished by touching the screen to manipulate smart devices. 


\section{Issues in Information Systems \\ Volume 17, Issue III, pp. 11-19, 2016}

In addition, the authorization of smart device functionality should be variable for different users. The full functionality or portions thereof should depend on the user's authorization level. The authority of accessibility measures the ability to manipulate smart devices, and the same device may require different authority from different users. The authority should be set up initially as part of the installation of the smart home services and this authority can only be changed by users with a higher authority. It is essential to build a secure intelligent system by offering different authorities. First of all, restricted access to some electronic appliances can prevent children from misusing them. Limiting the access to devices is an effective method to help keep children out of danger. Certain appliances such as stoves, microwaves, and washing machines should be operated only by adults, and when these devices are manipulated manually, the users with the appropriate authority should be notified and allowed to activate operations to take over the device. In addition, it is important to simplify the functionality of some smart devices and improve the user experience for children by reducing the utility of some devices and services. It may be inappropriate to display every function of certain smart devices to users with a basic level of access authority. In order to improve user experience, the design of the user interface should focus on the main functions of smart devices. For instance, users with a basic level of authority to the TV should only be able to manipulate functions such as power, channels, and volume and they should not have access to reset the system back to the factory settings. The different authorization levels can be established by granting different ranges of communication frequencies allowed. Users who operate various smart facilities as an administrator should have access to all kinds of information communication frequencies, while those who have basic authorization to smart devices can only access parts of the communication frequencies. This will restrict the access of users to some smart devices. Moreover, we can use passwords to set limitations on some users with respect to a particular smart device. The password such as a series of characters or a unique gesture can be created by users with the highest authority, and the password can only be deactivated by those who created it.

\section{Secure Privacy}

With the development of information technology, users must share more personal information than ever before in order to become part of the modern technology-driven society. However, our confidence in the security of our personal data hardly increases, and in fact may decrease, with the advancement of the technology. Many security issues still need to be solved and the quality of user experience can be negatively affected by inadvertent information leaks. In a smart home, users' privacy concerns a variety of data and actions including contact information, online search history, the settings of different smart devices, and users' lifestyle. It is important to realize that every action related to HCI can be monitored and recorded by advanced smart devices, which can be a double-edged sword. On the one hand, the smart devices can repeat the same operation without user's input, which allows the user to manipulate smart devices with a simple click or to continue an unfinished process. It is essential for smart facilities to keep the user's information for the purpose of simplifying operations as well as when the device or system is acting as a reminder. Monitoring the user's behaviors also plays a significant role in enhancing the efficiency of manipulating smart devices and contributing to the overall improvement of HCI. On the other hand, the user's behaviors are likely recorded and stored in the database, which can make the user's privacy vulnerable if the database is hacked. It is very important to keep the user's information safe. There may be some commercial entities that will attempt to make use of user information to provide advertisements and promotions. The user experience may be degraded by unexpected notifications and advertisements. Furthermore, this kind of unwanted communication can erode the user's level of confidence in the HCI.

In order to increase the security of the user's information, and to avoid the interruption of the user experience, the following mechanisms should be established. First, users should be able to choose if they want their smart devices to record their information or actions by allowing users to select their own preferences. There should be a check box for users to select whether they want their data and behaviors to be recorded or whether they want to join the improvement program helping smart home companies to collect the user's data. The agreement related to terms and conditions should be provided along with the check box. In addition, the user's data and behavior records should be stored in several databases. The information should be distributed among different databases in order to reduce the exposure of information when one of the databases is hacked. Users should also be able to choose to clear the access history or records automatically or manually, and users can choose to access databases by entering a password or identifying finger prints. Finally, smart home companies should provide some security measures. For example, antispy software and firewalls should be installed in every smart facility. Users can decide whether to buy these 


\section{Issues in Information Systems \\ Volume 17, Issue III, pp. 11-19, 2016}

software applications and if users purchase them, smart home companies should share the responsibility when the user's personal information is disclosed. Users can also purchase insurance not only for their smart devices, but also for the security of their personal information. Users and smart home companies should join forces to provide a comfortable and secure environment.

\section{DISCUSSION AND CONCLUSION}

The theory of the Internet of Things along with the current state of advanced technology provides a solid foundation on which to establish and improve the smart home. The smart home can be used to improve the quality of life of people with special needs. Smart homes, through their use of monitoring and optimizing smart devices, can also reduce the consumption of energy. Furthermore, the design of the HCI plays an increasingly important role in improving the smart home. The advancement of critical technologies such as sensors, monitors, and controllers provides the tools necessary to realize a more effective and efficient user experience.

Based on previous research and analysis, as well as the fundamental theory and practice of the smart home, we believe that a focus on improving the design of the HCI is important in order to enhance the user experience in the smart home. It is therefore essential to propose some approaches in order to produce efficient and effective HCI designs in the smart home. As such, we propose four potential approaches including efficient general control panels, effective user interfaces, variable accessibility, and secure privacy in order to significantly improve the design of the $\mathrm{HCI}$ and ultimately, the overall user experience.

First of all, the four approaches discussed in this paper provide general guidance regarding what should be expected in a smart home. Users need to become familiar with control panels and be aware of the capabilities of the user interface. The configurability of user access to smart device features should also be considered due to the fact that some users may need more functionality than the others. It is also very important to protect children by establishing authorization limits. Finally, the four approaches provide some useful guidelines that may assist designers and developers who wish to design and develop efficient and effective HCI in the smart home. For example, voice control for the manipulation of smart devices by the disabled.

Immediate future research of the smart home should try to validate the proposed concepts with the general public, i.e., users. Other fundamental components of the smart home such as security-related issues and entertainmentassociated topics should also be addressed. Firstly, a backup power source that can work independently when blackout happens should be set up in a smart home for the general monitor and alarm system. In addition, there should always be a secondary lock system in case of inaccurate reads of the sensors. Furthermore, the electrical radiation should be monitored and controlled within the safe range and all electronic appliances should be waterproof. In terms of smart home entertainment, further study need to be focused on a well-designed modularized structure, which makes a smart home changeable without breaking the foundation of the entire building. Moreover, the input and output ports of all smart home appliances should be identical or compatible. From the use of secure cameras and temperature monitors to remotely controlling TV and air-conditioner with an app on mobile devices such as an iPhone or an iPad, the concepts of smart home are becoming increasingly acceptable by the general public. We believe that the smart home will become a necessity in our lives in the near future.

In conclusion, the development of technology has made great strides in the realization of the smart home. An efficient and effective smart home cannot be achieved without the use of advanced technology. However, the seemingly unbounded requirements of smart home users will continue to present new technological challenges. This is a virtuous cycle. While it is difficult to predict the future direction of research and user requirements in this field, we wish to present some possible challenges for the future smart home. First, with the advent of 3D modeling, the future smart home could produce its own smart devices. Users could use a smart home 3D printer to produce new smart devices or repair damaged facilities within the smart home. In addition, a hologram could be employed as the basis for a user interface used to interact with smart devices. Furthermore, a movable smart wall could be used to modify the structure of the smart home on demand. Smart devices such as screens, speakers, air conditioners, and lights could be integrated into the "smart wall" to save space. 


\section{REFERENCES}

Bouchard, K., Bouchard, B., \& Bouzouane, A. (2012). Guidelines to efficient smart home design for rapid AI prototyping: A case study. Proceedings of the 5th International Conference on Pervasive Technologies Related to Assistive Environments (pp. 1-8), Haraklion, Crete, Greece.

Chan, M., Campo, E., Estève, D., \& Fourniols, J. (2009). Smart homes - Current features and future perspectives. Maturitas, 64(2), 90-97.

Chen, S., Chang, S., \& Chang, Y. (2010). Exploring a designer-oriented computer aided design interface for smart home device. Computer-Aided Design \& Applications, 7(6), 875-888.

Choi, E., Kwon, S., Lee, D., Lee, H., \& Chung, M. L. (2014). Towards successful user interaction with systems: Focusing on user-derived gestures for smart home systems. Applied Ergonomics, 45(4), 1196-1207.

Davidoff, S., Lee, M. K., Yiu, C., Zimmerman, J., \& Dey, A. K. (2006). Principles of smart home control. Ubicomp, 4206, 19-34.

De Silva, L. C., Morikawa, C., \& Petra, I. M. (2012). State of the art of smart homes. Engineering Applications of Artificial Intelligence, 25(7), 1313-1321.

Du, K., Wang, Z., \& Hong, M. (2013). Human machine interactive system on smart home of IoT. Journal of China Universities of Posts and Telecommunications, 20(1), 96-99.

Harper, R. (2003). Inside the smart home. London: Springer.

Kühnel, C., Westermann, T., Hemmert, F., Kratz, S., Müller, A., \& Möller, S. (2011). I'm home: Defining and evaluating a gesture set for smart-home control. International Journal of Human-Computer Studies, 69(11), 693-704.

Le, T., Reeder, B., Chung, J., Thompson, H., \& Demiris, G. (2014). Design of smart home sensor visualizations for older adults. Technology and Health Care, 22(4), 657-666.

Orpwood, R., Gibbs, C., Adlam, T., Faulkner, R., \& Meegahawatte, D. (2005). The design of smart homes for people with dementia - user-interface aspects. Universal Access in the Information Society, 4(2), 156-164.

Rashidi, P., \& Cook, D. J. (2009). Keeping the resident in the loop: Adapting the smart home to the user. IEEE Transactions on Systems, Man, and Cybernetics, Part A: Systems and Humans, 39(5), 1-11.

Roe, P. R. W. (2007). Towards an inclusive future. Brussels: COST.

Saffer, D. (2009). Designing gestural interfaces. Ottawa: O'Reilly Media.

Vega-Barbas, M., Pau, I., Martin-Ruiz, M. L., \& Seoane, F. (2015). Adaptive software architecture based on confident HCI for the deployment of sensitive services in smart homes. Sensors, 15(4), 7295-7320.

Wilson, C., Hargreaves, T., \& Hauxwell-Baldwin, R. (2015). Smart homes and their users: A systematic analysis and key challenges. Personal and Ubiquitous Computing, 19(2), 463-476.

Zhang, B., Rau, P. P., \& Salvendy, G. (2009). Design and evaluation of smart home user interface: Effects of age, tasks and intelligence level. Behaviour \& Information Technology, 28(3), 239-249.

Zimmermann, G., Henka, A., Strobbe, C., Mack, S., \& Landmesser, A. (2013). Towards context-driven user interfaces in smart homes. Proceedings of the Sixth International Conference on Advances in Humanoriented and Personalized Mechanisms, Technologies, and Services (pp. 98-103), Venice, Italy. 